\title{
CONFORMAL AND PARACONTACTLY GEODESIC TRANSFORMATIONS OF ALMOST PARACONTACT METRIC STRUCTURES
}

\author{
Adara M. Blaga
}

(c) 2020 by University of Niš, Serbia | Creative Commons License: CC BY-NC-ND

\begin{abstract}
We give the expressions of the virtual and the structure tensor fields of an almost paracontact metric structure. We also introduce the notion of paracontactly geodesic transformation and prove that the structure tensor field is invariant under conformal and paracontactly geodesic transformations. For the particular case of paraKenmotsu structure, we give a necessary and sufficient condition for a conformal transformation to map it to an $\alpha$-para-Kenmotsu structure and show that a para-Kenmotsu manifold admits no nontrivial paracontactly geodesic transformation of the metric. In the conformal case, the virtual tensor field is invariant.

Keywords: tensor field, paracontact metric structure, geodesic transformation.
\end{abstract}

\section{Introduction}

Let $M$ be a $(2 n+1)$-dimensional smooth manifold, $\varphi$ a (1,1)-tensor field called the structure endomorphism, $\xi$ a vector field called the characteristic vector field, $\eta$ a 1-form called the contact form and $g$ a pseudo-Riemannian metric on $M$. In this case, we say that $(\varphi, \xi, \eta, g)$ defines an almost paracontact metric structure on $M$ [2] if

1. $\varphi^{2} X=X-\eta(X) \xi$, for any $X \in \chi(M)$;

2. $\eta(\xi)=1$;

3. $g(\varphi X, \varphi Y)=-g(X, Y)+\eta(X) \eta(Y)$, for any $X, Y \in \chi(M)$

and $\varphi$ induces on the $2 n$-dimensional distribution $\operatorname{ker} \eta$ an almost paracomplex structure $P$ and the eigensubbundles corresponding to the eigenvalues 1 and -1 of $P$, respectively, have equal dimension $n$.

From the definition, it follows that $\varphi \xi=0, \eta(\varphi X)=0, \eta(X)=g(X, \xi), g(\xi, \xi)=$ $1, g(\varphi X, Y)=-g(X, \varphi Y)$, for any $X, Y \in \chi(M)$ and $\operatorname{ker} \varphi^{2}=\operatorname{ker} \varphi$.

Received December 22, 2018; accepted January 16, 2019

2010 Mathematics Subject Classification. Primary 53B05, 53D35; Secondary 70G45 


\section{The virtual and the structure tensor fields}

Consider $B_{\nabla}^{\varphi}(X, Y):=\frac{1}{2} \varphi\left(\left(\nabla_{\varphi X} \varphi\right) \varphi Y+\varphi\left(\left(\nabla_{\varphi^{2} X} \varphi\right) \varphi Y\right)\right)$ the virtual and $C_{\nabla}^{\varphi}(X, Y):=\frac{1}{2} \varphi\left(\left(\nabla_{\varphi X} \varphi\right) \varphi Y-\varphi\left(\left(\nabla_{\varphi^{2} X} \varphi\right) \varphi Y\right)\right)$ the structure tensor fields of the almost paracontact metric structure $(\varphi, \xi, \eta, g)$ which is connected to the Nijenhuis tensor field of $\varphi$ used in studying the normality of the structure.

Proposition 2.1. The virtual and the structure tensor fields of the almost paracontact metric structure $(\varphi, \xi, \eta, g)$ have the following properties:

1. $\varphi\left(B_{\nabla}^{\varphi}(X, Y)\right)=B_{\nabla}^{\varphi}(\varphi X, Y)=-B_{\nabla}^{\varphi}(X, \varphi Y)$;

2. $B_{\nabla}^{\varphi}(\varphi X, \varphi Y)=-B_{\nabla}^{\varphi}(X, Y)$;

3. $g\left(B_{\nabla}^{\varphi}(X, Y), Z\right)+g\left(Y, B_{\nabla}^{\varphi}(X, Z)\right)=0$;

4. $\varphi\left(C_{\nabla}^{\varphi}(X, Y)\right)=-C_{\nabla}^{\varphi}(\varphi X, Y)=-C_{\nabla}^{\varphi}(X, \varphi Y)$;

5. $C_{\nabla}^{\varphi}(\varphi X, \varphi Y)=C_{\nabla}^{\varphi}(X, Y)$;

6. $g\left(C_{\nabla}^{\varphi}(X, Y), Z\right)+g\left(Y, C_{\nabla}^{\varphi}(X, Z)\right)=0$,

for any $X, Y, Z \in \chi(M)$.

Proof. Notice that

$$
\begin{gathered}
B_{\nabla}^{\varphi}(X, Y):=\frac{1}{2} \varphi\left(\nabla_{\varphi X} \varphi^{2} Y-\varphi\left(\nabla_{\varphi X} \varphi Y\right)+\varphi\left(\nabla_{\varphi^{2} X} \varphi^{2} Y-\varphi\left(\nabla_{\varphi^{2} X} \varphi Y\right)\right)\right)= \\
=\frac{1}{2}\left[\varphi\left(\nabla_{\varphi X} \varphi^{2} Y\right)-\varphi^{2}\left(\nabla_{\varphi X} \varphi Y\right)+\varphi^{2}\left(\nabla_{\varphi^{2} X} \varphi^{2} Y\right)-\varphi^{3}\left(\nabla_{\varphi^{2} X} \varphi Y\right)\right] .
\end{gathered}
$$

We have:

$$
\begin{aligned}
\varphi\left(B_{\nabla}^{\varphi}(X, Y)\right) & =\frac{1}{2}\left[\varphi^{2}\left(\nabla_{\varphi X} \varphi^{2} Y\right)-\varphi^{3}\left(\nabla_{\varphi X} \varphi Y\right)+\varphi^{3}\left(\nabla_{\varphi^{2} X} \varphi^{2} Y\right)-\varphi^{4}\left(\nabla_{\varphi^{2} X} \varphi Y\right)\right] \\
B_{\nabla}^{\varphi}(\varphi X, Y) & =\frac{1}{2}\left[\varphi\left(\nabla_{\varphi^{2} X} \varphi^{2} Y\right)-\varphi^{2}\left(\nabla_{\varphi^{2} X} \varphi Y\right)+\varphi^{2}\left(\nabla_{\varphi^{3} X} \varphi^{2} Y\right)-\varphi^{3}\left(\nabla_{\varphi^{3} X} \varphi Y\right)\right], \\
B_{\nabla}^{\varphi}(X, \varphi Y) & =\frac{1}{2}\left[\varphi\left(\nabla_{\varphi X} \varphi^{3} Y\right)-\varphi^{2}\left(\nabla_{\varphi X} \varphi^{2} Y\right)+\varphi^{2}\left(\nabla_{\varphi^{2} X} \varphi^{3} Y\right)-\varphi^{3}\left(\nabla_{\varphi^{2} X} \varphi^{2} Y\right)\right], \\
B_{\nabla}^{\varphi}(\varphi X, \varphi Y) & =\frac{1}{2}\left[\varphi\left(\nabla_{\varphi^{2} X} \varphi^{3} Y\right)-\varphi^{2}\left(\nabla_{\varphi^{2} X} \varphi^{2} Y\right)+\varphi^{2}\left(\nabla_{\varphi^{3} X} \varphi^{3} Y\right)-\varphi^{3}\left(\nabla_{\varphi^{3} X} \varphi^{2} Y\right)\right] .
\end{aligned}
$$

Because $\varphi^{3}=\varphi$ and $\varphi^{4}=\varphi^{2}$ it follows $\varphi\left(B_{\nabla}^{\varphi}(X, Y)\right)=B_{\nabla}^{\varphi}(\varphi X, Y)=-B_{\nabla}^{\varphi}(X, \varphi Y)$ and $B_{\nabla}^{\varphi}(\varphi X, \varphi Y)=-B_{\nabla}^{\varphi}(X, Y)$.

Similarly we can show $\varphi\left(C_{\nabla}^{\varphi}(X, Y)\right)=-C_{\nabla}^{\varphi}(\varphi X, Y)=-C_{\nabla}^{\varphi}(X, \varphi Y)$ and $C_{\nabla}^{\varphi}(\varphi X, \varphi Y)=C_{\nabla}^{\varphi}(X, Y)$. 
Taking into account that $g(\varphi X, Y)=-g(X, \varphi Y)$ and $g\left(\varphi^{2} X, \varphi^{2} Y\right)=-g(\varphi X, \varphi Y)$, for any $X, Y \in \chi(M)$, we get:

$$
\begin{aligned}
& \quad g\left(B_{\nabla}^{\varphi}(X, Y), Z\right)+g\left(Y, B_{\nabla}^{\varphi}(X, Z)\right)= \\
& =\frac{1}{2}\left[-g\left(\nabla_{\varphi X} \varphi^{2} Y, \varphi Z\right)-g\left(\nabla_{\varphi X} \varphi Y, \varphi^{2} Z\right)+g\left(\nabla_{\varphi^{2} X} \varphi^{2} Y, \varphi^{2} Z\right)+g\left(\nabla_{\varphi^{2} X} \varphi Y, \varphi Z\right)-\right. \\
& \left.-g\left(\nabla_{\varphi X} \varphi^{2} Z, \varphi Y\right)-g\left(\nabla_{\varphi X} \varphi Z, \varphi^{2} Y\right)+g\left(\nabla_{\varphi^{2} X} \varphi^{2} Z, \varphi^{2} Y\right)+g\left(\nabla_{\varphi^{2} X} \varphi Z, \varphi Y\right)\right]= \\
& =\frac{1}{2}\left[-\varphi X\left(g\left(\varphi^{2} Y, \varphi Z\right)\right)-\varphi X\left(g\left(\varphi Y, \varphi^{2} Z\right)\right)+\varphi^{2} X\left(g\left(\varphi^{2} Y, \varphi^{2} Z\right)\right)+\varphi^{2} X(g(\varphi Y, \varphi Z))\right]=0 .
\end{aligned}
$$

Similarly we can show $g\left(C_{\nabla}^{\varphi}(X, Y), Z\right)+g\left(Y, C_{\nabla}^{\varphi}(X, Z)\right)=0$.

Let $m$ and $l$ be the complementary projectors on the tangent bundle of $M$, defined by:

$$
m:=\eta \otimes \xi, \quad l:=I-\eta \otimes \xi
$$

and denoted by $\mathfrak{M}:=\operatorname{Im}(m)$ and $\mathfrak{L}:=\operatorname{Im}(l)$ (obviously, $l=\varphi^{2}$ ). Then $T M=$ $\mathfrak{M} \oplus \mathfrak{L}$, and from the properties of the almost paracontact metric structure it follows that $\mathfrak{M}=\operatorname{ker} \varphi$ and $\mathfrak{L}=\operatorname{ker} \eta$.

By $N_{\varphi}$, we denoted the Nijenhuis tensor field of $\varphi$ :

$$
\begin{gathered}
N_{\varphi}(X, Y):=\varphi^{2}[X, Y]+[\varphi X, \varphi Y]-\varphi[\varphi X, Y]-\varphi[X, \varphi Y]= \\
=\varphi^{2}\left(\nabla_{X} Y\right)-\varphi^{2}\left(\nabla_{Y} X\right)+\nabla_{\varphi X} \varphi Y-\nabla_{\varphi Y} \varphi X- \\
-\varphi\left(\nabla_{\varphi X} Y\right)+\varphi\left(\nabla_{Y} \varphi X\right)-\varphi\left(\nabla_{X} \varphi Y\right)+\varphi\left(\nabla_{\varphi Y} X\right) .
\end{gathered}
$$

Proposition 2.2. If $(\varphi, \xi, \eta, g)$ is an almost paracontact metric structure on $M$, then $\varphi^{2}\left(N_{\varphi}(\varphi X, \varphi Y)\right)=2\left(C_{\nabla}^{\varphi}(Y, X)-C_{\nabla}^{\varphi}(X, Y)\right)$, for any $X, Y \in \chi(M)$.

Proof. We have:

$$
\begin{gathered}
\varphi^{2}\left(N_{\varphi}(\varphi X, \varphi Y)\right)=\varphi^{2}\left(\varphi^{2}\left(\nabla_{\varphi X} \varphi Y\right)-\varphi^{2}\left(\nabla_{\varphi Y} \varphi X\right)+\nabla_{\varphi^{2} X} \varphi^{2} Y-\nabla_{\varphi^{2} Y} \varphi^{2} X-\right. \\
\left.-\varphi\left(\nabla_{\varphi^{2} X} \varphi Y\right)+\varphi\left(\nabla_{\varphi Y} \varphi^{2} X\right)-\varphi\left(\nabla_{\varphi X} \varphi^{2} Y\right)+\varphi\left(\nabla_{\varphi^{2} Y} \varphi\right)\right)= \\
=\varphi^{4}\left(\nabla_{\varphi X} \varphi Y\right)-\varphi^{4}\left(\nabla_{\varphi Y} \varphi X\right)+\varphi^{2}\left(\nabla_{\varphi^{2} X} \varphi^{2} Y\right)-\varphi^{2}\left(\nabla_{\varphi^{2} Y} \varphi^{2} X\right)- \\
-\varphi^{3}\left(\nabla_{\varphi^{2} X} \varphi Y\right)+\varphi^{3}\left(\nabla_{\varphi Y} \varphi^{2} X\right)-\varphi^{3}\left(\nabla_{\varphi X} \varphi^{2} Y\right)+\varphi^{3}\left(\nabla_{\varphi^{2} Y} \varphi\right) .
\end{gathered}
$$

Because $\varphi^{3}=\varphi$ and $\varphi^{4}=\varphi^{2}$ it follows

$$
\begin{gathered}
\varphi^{2}\left(N_{\varphi}(\varphi X, \varphi Y)\right)=\varphi^{2}\left(\nabla_{\varphi X} \varphi\right)-\varphi^{2}\left(\nabla_{\varphi Y} \varphi X\right)+\varphi^{2}\left(\nabla_{\varphi^{2} X} \varphi^{2} Y\right)-\varphi^{2}\left(\nabla_{\varphi^{2} Y} \varphi^{2} X\right)- \\
-\varphi\left(\nabla_{\varphi^{2} X} \varphi\right)+\varphi\left(\nabla_{\varphi Y} \varphi^{2} X\right)-\varphi\left(\nabla_{\varphi X} \varphi^{2} Y\right)+\varphi\left(\nabla_{\varphi^{2} Y} \varphi\right)= \\
=-\left[\varphi\left(\nabla_{\varphi X} \varphi^{2} Y\right)-\varphi^{2}\left(\nabla_{\varphi X} \varphi Y\right)-\varphi^{2}\left(\nabla_{\varphi^{2} X} \varphi^{2} Y\right)+\varphi\left(\nabla_{\varphi^{2} X} \varphi\right)\right]+ \\
+\left[\varphi\left(\nabla_{\varphi Y} \varphi^{2} X\right)-\varphi^{2}\left(\nabla_{\varphi Y} \varphi X\right)-\varphi^{2}\left(\nabla_{\varphi^{2} Y} \varphi^{2} X\right)+\varphi\left(\nabla_{\varphi^{2} Y} \varphi\right)\right]= \\
=-2 C_{\nabla}^{\varphi}(X, Y)+2 C_{\nabla}^{\varphi}(Y, X) .
\end{gathered}
$$


Corollary 2.1. Let $(\varphi, \xi, \eta, g)$ be an almost paracontact metric structure on $M$.

1. If the Nijenhuis tensor field of $\varphi$ vanishes identically, then the structure tensor field is symmetric.

2. If the structure tensor field is symmetric, then $N_{\varphi}(\varphi X, \varphi Y) \in \mathfrak{M}$, for any $X$, $Y \in \chi(M)$.

Proof. From Proposition 2.2 we have for any $X, Y \in \chi(M)$ :

$$
\varphi^{2}\left(N_{\varphi}(\varphi X, \varphi Y)\right)=2\left(C_{\nabla}^{\varphi}(Y, X)-C_{\nabla}^{\varphi}(X, Y)\right) .
$$

1. If $N_{\varphi}=0$ follows $C_{\nabla}^{\varphi}(Y, X)-C_{\nabla}^{\varphi}(X, Y)=0$, for any $X, Y \in \chi(M)$.

2. If $C_{\nabla}^{\varphi}(Y, X)=C_{\nabla}^{\varphi}(X, Y)$, for any $X, Y \in \chi(M)$ follows $\varphi^{2}\left(N_{\varphi}(\varphi X, \varphi Y)\right)=0$ i.e. $N_{\varphi}(\varphi X, \varphi Y) \in \operatorname{ker} \varphi^{2}=\operatorname{ker} \varphi=\mathfrak{M}$.

\section{Paracontactly geodesic transformations}

We will introduce the notion of paracontactly geodesic transformation of an almost paracontact metric structure and study the invariance of the virtual and structure tensor fields under paracontactly geodesic transformations.

Recall that a diffeomorphism between two pseudo-Riemannian manifolds $\Phi$ : $(M, g) \rightarrow(\bar{M}, \bar{g})$ is called geodesic map, if it takes each geodesic of $(M, g)$ to a geodesic of $(\bar{M}, \bar{g})$. In this case, the pseudo-Riemannian metric $\tilde{g}:=\Phi^{*} \bar{g}$ on $M$ is called geodesic transformation of $g$. Note that the metrics $g$ and $\tilde{g}$ have common geodesics.

Let $(\varphi, \xi, \eta, g)$ be an almost paracontact metric structure on the smooth manifold $M$. Then:

Definition 3.1. A geodesic transformation $g \rightarrow \tilde{g}$ of the pseudo-Riemannian metric $g$ on $M$ is called paracontactly geodesic transformation if $(\varphi, \xi, \eta, \tilde{g})$ is also an almost paracontact metric structure on $M$.

A simple example similar like in the almost contact case [3] is the following.

Example 3.1. Let $\Phi:(M, \varphi, \xi, \eta, g) \rightarrow(\bar{M}, \bar{\varphi}, \bar{\xi}, \bar{\eta}, \bar{g})$ be a geodesic map preserving the almost paracontact structure, that is, $\bar{\varphi}=\Phi_{*} \circ \varphi \circ\left(\Phi_{*}\right)^{-1}, \bar{\xi}=\Phi_{*}(\xi), \bar{\eta}=\left(\Phi^{*}\right)^{-1} \eta$. Then $g \rightarrow \tilde{g}:=\Phi^{*} \bar{g}$ is a paracontactly geodesic transformation of $g$ on $M$.

Let $(\varphi, \xi, \eta, g)$ be an almost paracontact metric structure on the smooth manifold $M$ and $g \rightarrow \tilde{g}$ a paracontactly geodesic transformation of $g$.

It was proved [5] that the tensor $T$ of the affine deformation from the Levi-Civita connection $\nabla$ of $g$ to the Levi-Civita connection $\tilde{\nabla}$ of $\tilde{g}$ has the form $T(X, Y):=$ $\tilde{\nabla}_{X} Y-\nabla_{X} Y=\psi(X) Y+\psi(Y) X, X, Y \in \chi(M)$, for $\psi$ an exact 1-form on $M$ called the 1-form of geodesic distortion. In this case, the Levi-Civita connections associated to $g$ and $\tilde{g}$ satisfy $\left(\tilde{\nabla}_{X} \varphi\right) Y=\left(\nabla_{X} \varphi\right) Y+\psi(\varphi Y) X-\psi(Y) \varphi X$, for any $X, Y \in \chi(M)$. 
Proposition 3.1. The virtual and the structure tensor fields of the transformed structure have the following properties:

1. $B_{\tilde{\nabla}}^{\varphi}(X, Y)=B_{\nabla}^{\varphi}(X, Y)+\psi\left(\varphi^{2} Y\right) \varphi^{2} X-\psi(\varphi Y) \varphi X$

2. $C_{\tilde{\nabla}}^{\varphi}(X, Y)=C_{\nabla}^{\varphi}(X, Y)$,

for any $X, Y \in \chi(M)$.

Proof.

$$
\begin{gathered}
B_{\tilde{\nabla}}^{\varphi}(X, Y):=\frac{1}{2} \varphi\left(\left(\tilde{\nabla}_{\varphi X} \varphi\right) \varphi Y+\varphi\left(\left(\tilde{\nabla}_{\varphi^{2} X} \varphi\right) \varphi Y\right)\right)= \\
=\frac{1}{2} \varphi\left(\left(\nabla_{\varphi X} \varphi\right) \varphi Y+\psi\left(\varphi^{2} Y\right) \varphi X-\psi(\varphi Y) \varphi^{2} X+\right. \\
\left.+\varphi\left(\left(\nabla_{\varphi^{2} X} \varphi\right) \varphi Y+\psi\left(\varphi^{2} Y\right) \varphi^{2} X-\psi(\varphi Y) \varphi^{3} X\right)\right):= \\
:=B_{\nabla}^{\varphi}(X, Y)+\frac{1}{2}\left[\psi\left(\varphi^{2} Y\right) \varphi^{2} X-\psi(\varphi Y) \varphi^{3} X+\psi\left(\varphi^{2} Y\right) \varphi^{4} X-\psi(\varphi Y) \varphi^{5} X\right] .
\end{gathered}
$$

Because $\varphi^{3}=\varphi, \varphi^{4}=\varphi^{2}$ and $\varphi^{5}=\varphi$, we get the first relation. The second one can be similarly obtained.

We can therefore state the theorem:

Theorem 3.1. The structure tensor field of an almost paracontact metric structure is invariant under paracontactly geodesic transformations.

Concerning the virtual tensor, we give necessary and sufficient conditions for it to be invariant.

Theorem 3.2. The virtual tensor field of the almost paracontact metric structure $(\varphi, \xi, \eta, g)$ is invariant under paracontactly geodesic transformations $g \rightarrow \tilde{g}$ (i.e. $\left.B_{\tilde{\nabla}}^{\varphi}=B_{\nabla}^{\varphi}\right)$ if and only if $\psi\left(\varphi^{2} Y\right) \varphi X-\psi(\varphi Y) X \in \mathfrak{M}$, for any $X, Y \in \chi(M)$.

Proof. From Proposition 3.1, the condition $B_{\tilde{\nabla}}^{\varphi}=B_{\nabla}^{\varphi}$ is equivalent to $0=\psi\left(\varphi^{2} Y\right) \varphi^{2} X-\psi(\varphi Y) \varphi X=\varphi\left[\psi\left(\varphi^{2} Y\right) \varphi X-\psi(\varphi Y) X\right]$, for any $X, Y \in \chi(M)$.

\section{Conformal transformations}

By a conformal transformation of the almost paracontact metric structure $(\varphi, \xi, \eta, g)$ we understand the passage to the almost paracontact metric structure $(\varphi, \tilde{\xi}, \tilde{\eta}, \tilde{g})$, where $\tilde{\xi}:=e^{f} \xi, \tilde{\eta}:=e^{-f} \eta, \tilde{g}:=e^{-2 f} g$, for $f$ a smooth function on the manifold $M$. In the part to follow, we shall study the invariance of the virtual and the structure tensor fields under conformal transformations.

Because the Levi-Civita connection $\tilde{\nabla}$ of $\tilde{g}$ and $\nabla$ of $g$ satisfy $\tilde{\nabla}_{X} Y=\nabla_{X} Y-$ $\left[X(f) Y+Y(f) X-g(X, Y) \operatorname{grad}_{g}(f)\right]$ it follows $\left(\tilde{\nabla}_{X} \varphi\right) Y=\left(\nabla_{X} \varphi\right) Y-d f(\varphi Y) X+$ $d f(Y) \varphi X+g(X, \varphi Y) \operatorname{grad}_{g}(f)-g(X, Y) \varphi\left(\operatorname{grad}_{g}(f)\right)$, for any $X, Y \in \chi(M)$. 
Proposition 4.1. The virtual and the structure tensor fields of the transformed structure have the following properties:

1. $B_{\tilde{\nabla}}^{\varphi}(X, Y)=B_{\nabla}^{\varphi}(X, Y)-d f\left(\varphi^{2} Y\right) \varphi^{2} X+d f(\varphi Y) \varphi X-g(\varphi X, \varphi Y) \varphi^{2}\left(\operatorname{grad}_{g}(f)\right)+$ $g(\varphi X, Y) \varphi\left(\operatorname{grad}_{g}(f)\right)$;

2. $C_{\tilde{\nabla}}^{\varphi}(X, Y)=C_{\nabla}^{\varphi}(X, Y)$,

for any $X, Y \in \chi(M)$.

Proof.

$$
\begin{gathered}
B_{\tilde{\nabla}}^{\varphi}(X, Y):=\frac{1}{2} \varphi\left(\left(\tilde{\nabla}_{\varphi X} \varphi\right) \varphi Y+\varphi\left(\left(\tilde{\nabla}_{\varphi^{2} X} \varphi\right) \varphi Y\right)\right)= \\
=\frac{1}{2} \varphi\left(\left(\nabla_{\varphi X} \varphi\right) \varphi Y-d f\left(\varphi^{2} Y\right) \varphi X+d f(\varphi Y) \varphi^{2} X+g\left(\varphi X, \varphi^{2} Y\right) \operatorname{grad}_{g}(f)-\right. \\
-g(\varphi X, \varphi Y) \varphi\left(\operatorname{grad}_{g}(f)\right)+\varphi\left(\left(\nabla_{\varphi^{2} X} \varphi\right) \varphi Y-d f\left(\varphi^{2} Y\right) \varphi^{2} X+d f(\varphi Y) \varphi^{3} X+\right. \\
\left.\left.+g\left(\varphi^{2} X, \varphi^{2} Y\right) \operatorname{grad}_{g}(f)-g\left(\varphi^{2} X, \varphi Y\right) \varphi\left(\operatorname{grad}_{g}(f)\right)\right)\right):= \\
:=B_{\nabla}^{\varphi}(X, Y)+\frac{1}{2}\left[-d f\left(\varphi^{2} Y\right) \varphi^{2} X+d f(\varphi Y) \varphi^{3} X+g\left(\varphi X, \varphi^{2} Y\right) \varphi\left(\operatorname{grad}_{g}(f)\right)-\right. \\
-g(\varphi X, \varphi Y) \varphi^{2}\left(\operatorname{grad}_{g}(f)\right)-d f\left(\varphi^{2} Y\right) \varphi^{4} X+d f(\varphi Y) \varphi^{5} X+ \\
\left.+g\left(\varphi^{2} X, \varphi^{2} Y\right) \varphi^{2}\left(\operatorname{grad}_{g}(f)\right)-g\left(\varphi^{2} X, \varphi Y\right) \varphi^{3}\left(\operatorname{grad}_{g}(f)\right)\right] .
\end{gathered}
$$

Because $\varphi^{3}=\varphi, \varphi^{4}=\varphi^{2}$ and $\varphi^{5}=\varphi, g(\varphi X, Y)=-g(X, \varphi Y)$ and $g\left(\varphi^{2} X, \varphi^{2} Y\right)=$ $-g(\varphi X, \varphi Y)$, for any $X, Y \in \chi(M)$, we get the first relation. The second one can be similarly obtained.

We can therefore state the theorem:

Theorem 4.1. The structure tensor field of an almost paracontact metric structure is invariant under conformal transformations.

Concerning the virtual tensor, we shall find a necessary and sufficient condition for it to be also invariant. Note that in the particular case when $\tilde{g}=\lambda g$, for $\lambda$ a constant positive function, $B_{\tilde{\nabla}}^{\varphi}=B_{\nabla}^{\varphi}$.

Theorem 4.2. The virtual tensor field of the almost paracontact metric structure $(\varphi, \xi, \eta, g)$ is invariant under conformal transformations $g \rightarrow \tilde{g}:=e^{-2 f} g$ (i.e. $B_{\tilde{\nabla}}^{\varphi}=$ $\left.B_{\nabla}^{\varphi}\right)$ if and only if $\operatorname{grad}_{g}(f) \in \mathfrak{M}$.

Proof. We follow the steps used in proving an analogue result for the almost contact metric case [4]. Therefore, $B_{\tilde{\nabla}}^{\varphi}=B_{\nabla}^{\varphi}$ if and only if for any $X, Y \in \chi(M)$

$$
d f\left(\varphi^{2} Y\right) \varphi^{2} X-d f(\varphi Y) \varphi X=-g(\varphi X, \varphi Y) \varphi^{2}\left(\operatorname{grad}_{g}(f)\right)+g(\varphi X, Y) \varphi\left(\operatorname{grad}_{g}(f)\right)
$$


Changing the role of $X$ and $Y$ in the previous relation, we obtain:

$$
\begin{gathered}
d f\left(\varphi^{2} X\right) \varphi^{2} Y-d f(\varphi X) \varphi Y=-g(\varphi Y, \varphi X) \varphi^{2}\left(\operatorname{grad}_{g}(f)\right)+g(\varphi Y, X) \varphi\left(\operatorname{grad}_{g}(f)\right)= \\
=-g(\varphi Y, \varphi X) \varphi^{2}\left(\operatorname{grad}_{g}(f)\right)-g(Y, \varphi X) \varphi\left(\operatorname{grad}_{g}(f)\right)
\end{gathered}
$$

and adding the two relations we get:

$d f\left(\varphi^{2} Y\right) \varphi^{2} X-d f(\varphi Y) \varphi X+d f\left(\varphi^{2} X\right) \varphi^{2} Y-d f(\varphi X) \varphi Y=-2 g(\varphi X, \varphi Y) \varphi^{2}\left(\operatorname{grad}_{g}(f)\right)$.

Then for $Y:=X$ we obtain:

$$
d f\left(\varphi^{2} X\right) \varphi^{2} X-d f(\varphi X) \varphi X=-g(\varphi X, \varphi X) \varphi^{2}\left(\operatorname{grad}_{g}(f)\right) .
$$

If we assume that $g(X, X)=1$ it follows that $g(\varphi X, \varphi X)=-1+[\eta(X)]^{2}$ and if $X \in \mathfrak{L}$ we get $\eta(X)=0$ and $\varphi^{2} X=X$. Therefore, for $X \in \mathfrak{L}$ such that $g(X, X)=1$ we obtain $g(\varphi X, \varphi X)=-1$ and $\varphi^{2} X=X$. Then the equality becomes

$$
d f(X) X-d f(\varphi X) \varphi X=\varphi^{2}\left(\operatorname{grad}_{g}(f)\right),
$$

which means that $\varphi^{2}\left(\operatorname{grad}_{g}(f)\right)$ is a linear combination of the vector fields $X$ and $\varphi X$. Writing this for $X:=Y$ we get:

$$
d f(Y) Y-d f(\varphi Y) \varphi Y=\varphi^{2}\left(\operatorname{grad}_{g}(f)\right)
$$

and substracting the two relations

$$
d f(X) X-d f(\varphi X) \varphi X-d f(Y) Y+d f(\varphi Y) \varphi Y=0 .
$$

Since the four vector fields $\{X, \varphi X, Y, \varphi Y\}$ are linearly independent, we deduce that $d f(X)=0$ equivalent to $g\left(\operatorname{grad}_{g}(f), X\right)=0$, for $X \in \mathfrak{L}$, which means that $\operatorname{grad}_{g}(f) \in \mathfrak{L}^{\perp}=\mathfrak{M}$.

Then

Conversely, if $\operatorname{grad}_{g}(f) \in \mathfrak{M}=\operatorname{ker} \varphi$ we get $\varphi^{2}\left(\operatorname{grad}_{g}(f)\right)=\varphi\left(\operatorname{grad}_{g}(f)\right)=0$.

$$
\begin{gathered}
d f\left(\varphi^{2} Y\right) \varphi^{2} X-d f(\varphi Y) \varphi X=g\left(\operatorname{grad}_{g}(f), \varphi^{2} Y\right) \varphi^{2} X-g\left(\operatorname{grad}_{g}(f), \varphi Y\right) \varphi X= \\
=g\left(\varphi^{2}\left(\operatorname{grad}_{g}(f)\right), Y\right) \varphi^{2} X+g\left(\varphi\left(\operatorname{grad}_{g}(f)\right), Y\right) \varphi X=0
\end{gathered}
$$

and so $B_{\tilde{\nabla}}^{\varphi}=B_{\nabla}^{\varphi}$.

\section{Applications}

We shall consider the particular case of a para-Kenmotsu manifold. Let $\alpha$ be a smooth real function on the smooth manifold $M$.

Definition 5.1. [6] We say that the almost paracontact metric structure $(\varphi, \xi, \eta, g)$ is $\alpha$-para-Kenmotsu if the Levi-Civita connection $\nabla$ of $g$ satisfies $\left(\nabla_{X} \varphi\right) Y=\alpha[g(\varphi X, Y) \xi-\eta(Y) \varphi X]$, for any $X, Y \in \chi(M)$. 
For $\alpha \equiv 1$, we call $(\varphi, \xi, \eta, g)$ para-Kenmotsu structure.

Theorem 5.1. A conformal transformation with defining function $f$ maps a paraKenmotsu structure to an $e^{f}$-para-Kenmotsu structure if and only if $f$ is locally constant.

Proof. Let $(\varphi, \xi, \eta, g)$ be a para-Kenmotsu structure on $M$ and $(\varphi, \tilde{\xi}, \tilde{\eta}, \tilde{g})$ be a conformal transform of $(\varphi, \xi, \eta, g)$ with $\tilde{\xi}=e^{f} \xi, \tilde{\eta}=e^{-f} \eta, \tilde{g}=e^{-2 f} g$.

We know that:

$\left(\tilde{\nabla}_{X} \varphi\right) Y=\left(\nabla_{X} \varphi\right) Y-d f(\varphi Y) X+d f(Y) \varphi X+g(X, \varphi Y) \operatorname{grad}_{g}(f)-g(X, Y) \varphi\left(\operatorname{grad}_{g}(f)\right)$, for any $X, Y \in \chi(M)$.

If $(\varphi, \xi, \eta, g)$ is para-Kenmotsu structure, we have:

$$
\left(\nabla_{X} \varphi\right) Y=g(\varphi X, Y) \xi-\eta(Y) \varphi X
$$

for any $X, Y \in \chi(M)$. It follows:

$$
\begin{gathered}
\left(\tilde{\nabla}_{X} \varphi\right) Y=g(\varphi X, Y) \xi-\eta(Y) \varphi X-d f(\varphi Y) X+d f(Y) \varphi X+ \\
+g(X, \varphi Y) \operatorname{grad}_{g}(f)-g(X, Y) \varphi\left(\operatorname{grad}_{g}(f)\right),
\end{gathered}
$$

for any $X, Y \in \chi(M)$. Replacing $\xi=e^{-f} \tilde{\xi}, \eta=e^{f} \tilde{\eta}, g=e^{2 f} \tilde{g}$, we obtain:

$$
\begin{gathered}
\left(\tilde{\nabla}_{X} \varphi\right) Y=e^{f}[\tilde{g}(\varphi X, Y) \tilde{\xi}-\tilde{\eta}(Y) \varphi X]-d f(\varphi Y) X+d f(Y) \varphi X+ \\
+e^{2 f}\left[\tilde{g}(X, \varphi Y) \operatorname{grad}_{g}(f)-\tilde{g}(X, Y) \varphi\left(\operatorname{grad}_{g}(f)\right)\right],
\end{gathered}
$$

for any $X, Y \in \chi(M)$.

Then $(\varphi, \tilde{\xi}, \tilde{\eta}, \tilde{g})$ is $e^{f}$-para-Kenmotsu structure, i.e.

$$
\left(\tilde{\nabla}_{X} \varphi\right) Y=e^{f}[\tilde{g}(\varphi X, Y) \tilde{\xi}-\tilde{\eta}(Y) \varphi X],
$$

for any $X, Y \in \chi(M)$ if and only if

$$
d f(\varphi Y) X-d f(Y) \varphi X=e^{2 f}\left[\tilde{g}(X, \varphi Y) \operatorname{grad}_{g}(f)-\tilde{g}(X, Y) \varphi\left(\operatorname{grad}_{g}(f)\right)\right],
$$

for any $X, Y \in \chi(M)$. For $X:=\xi$ and $Y:=\xi$, because $\varphi \xi=0$, we get $\varphi\left(\operatorname{grad}_{g}(f)\right)=0$, i.e. $\operatorname{grad}_{g}(f) \in \operatorname{ker} \varphi=\mathfrak{M}$. Replacing this in the previous relation, we have:

$$
d f(\varphi Y) X-d f(Y) \varphi X=e^{2 f} \tilde{g}(X, \varphi Y) \operatorname{grad}_{g}(f),
$$

for any $X, Y \in \chi(M)$. For $Y:=\xi$, because $\varphi \xi=0$, we get $d f(\xi)=0$ which is equivalent with $g\left(\operatorname{grad}_{g}(f), \xi\right)=0$ and with $\operatorname{grad}_{g}(f) \perp \xi$, i.e. $\operatorname{grad}_{g}(f) \in \operatorname{ker} \eta=$ $\mathfrak{L}$.

Therefore, $\operatorname{grad}_{g}(f) \in \mathfrak{M} \cap \mathfrak{L}=\{0\}$, which means that $f$ is locally constant. 
Conversely, if $f$ is locally constant, then $d f=0, \operatorname{grad}_{g}(f)=0$ and

$$
\begin{gathered}
\left(\tilde{\nabla}_{X} \varphi\right) Y=e^{f}[\tilde{g}(\varphi X, Y) \tilde{\xi}-\tilde{\eta}(Y) \varphi X]-d f(\varphi Y) X+d f(Y) \varphi X+ \\
+e^{2 f}\left[\tilde{g}(X, \varphi Y) \operatorname{grad}_{g}(f)-\tilde{g}(X, Y) \varphi\left(\operatorname{grad}_{g}(f)\right)\right]= \\
=e^{f}[\tilde{g}(\varphi X, Y) \tilde{\xi}-\tilde{\eta}(Y) \varphi X],
\end{gathered}
$$

for any $X, Y \in \chi(M)$, i.e. $(\varphi, \tilde{\xi}, \tilde{\eta}, \tilde{g})$ is $e^{f}$-para-Kenmotsu structure.

Corollary 5.1. If a conformal transformation with defining function $f$ maps a para-Kenmotsu structure to an $e^{f}$-para-Kenmotsu one, then the virtual tensor field is invariant.

Proof. From Theorem 5.1 we have that $f$ is locally constant and from Proposition 4.1 we obtain $B_{\vec{\nabla}}^{\varphi}=B_{\nabla}^{\varphi}$.

Theorem 5.2. A para-Kenmotsu manifold admits no nontrivial paracontactly geodesic transformation of the metric.

Proof. Let $(\varphi, \xi, \eta, g)$ be a para-Kenmotsu structure on $M$ and $g \mapsto \tilde{g}$ be a paracontactly geodesic transformation with $\tilde{\nabla}_{X} Y=\nabla_{X} Y+\psi(X) Y+\psi(Y) X$, for $X$, $Y \in \chi(M)$.

We know that:

$$
\left(\tilde{\nabla}_{X} \varphi\right) Y=\left(\nabla_{X} \varphi\right) Y+\psi(\varphi Y) X-\psi(Y) \varphi X
$$

for any $X, Y \in \chi(M)$.

If $(\varphi, \xi, \eta, g)$ is para-Kenmotsu structure, we have:

$$
\left(\nabla_{X} \varphi\right) Y=g(\varphi X, Y) \xi-\eta(Y) \varphi X
$$

for any $X, Y \in \chi(M)$. It follows:

$$
\left(\tilde{\nabla}_{X} \varphi\right) Y=g(\varphi X, Y) \xi-\eta(Y) \varphi X+\psi(\varphi Y) X-\psi(Y) \varphi X,
$$

for any $X, Y \in \chi(M)$.

If the transformed structure $(\varphi, \xi, \eta, \tilde{g})$ would be para-Kenmotsu, too, then:

$$
\left(\tilde{\nabla}_{X} \varphi\right) Y=\tilde{g}(\varphi X, Y) \xi-\eta(Y) \varphi X
$$

for any $X, Y \in \chi(M)$ and replacing this in the previous relation, we obtain:

$$
[\tilde{g}(\varphi X, Y)-g(\varphi X, Y)] \xi=\psi(\varphi Y) X-\psi(Y) \varphi X,
$$

for any $X, Y \in \chi(M)$. For $X:=\xi$, because $\varphi \xi=0$, we get $\psi(\varphi Y)=0$, for any $Y \in \chi(M)$. It follows:

$$
[\tilde{g}(\varphi X, Y)-g(\varphi X, Y)] \xi=-\psi(Y) \varphi X
$$

for any $X, Y \in \chi(M)$ and applying $\varphi$, we get:

$$
\psi(Y) \varphi X=0
$$

for any $X, Y \in \chi(M)$, which implies $\psi=0$ or $\varphi=0$, that is impossible. Therefore, the paracontactly geodesic transformation $g \mapsto \tilde{g}$ can not map the para-Kenmotsu structure $(\varphi, \xi, \eta, g)$ to a para-Kenmotsu one. 


\section{R E F E R E N C E S}

1. A. M. Blaga, C. IDA: Generalized almost paracontact structures, An. Şt. Univ. Ovidius Constanţa 23, no. 1 (2015), 53-64.

2. S. ERDEM: On almost (para) contact (hyperbolic) metric manifolds and harmonicity of $\left(\varphi, \varphi^{\prime}\right)$-holomorphic maps between them, Houston Math. J. 28 (2002), 21-45.

3. V. F. Kirichenko, N. N. Dondukova: Contactly Geodesic Transformations of Almost-Contact Metric Structures, Math. Notes 80, no. 2 (2006), 204-213.

4. V. F. Kirichenko, I. V. Uskorev: Invariants of Conformal Transformations of Almost Contact Metric Structures, Math. Notes 84, no. 6 (2008), 783-794.

5. N. S. Sinyukov: Geodesic Maps of Riemannian Spaces, Nauka, Moskow, 1979.

6. J. WELYCZKO: Slant curves in 3-dimensional normal almost paracontact metric manifolds, Mediterr. J. Math. 11 (2014), 965-978.

Adara M. Blaga

Faculty of Mathematics and Computer Science

Department of Mathematics

Bd. V. Pârvan 4-6

300223 Timişoara, România

adarablaga@yahoo.com 